This is an electronic reprint of the original article. This reprint may differ from the original in pagination and typographic detail.

Author(s): Abdi Mahmoudaliloo, Younes; Ristaniemi, Tapani

Title: $\quad$ Extension of Deflection Coefficient for Linear Fusion of Quantized Reports in Cooperative Sensing

Year: $\quad 2014$

Version:

Please cite the original version:

Abdi Mahmoudaliloo, Y., \& Ristaniemi, T. (2014). Extension of Deflection Coefficient for Linear Fusion of Quantized Reports in Cooperative Sensing. In Proceedings of IEEE PIMRC 2014 : IEEE 25th International Symposium on Personal, Indoor and Mobile Radio Communications (pp. 928-932). IEEE. IEEE International Symposium on Personal, Indoor, and Mobile Radio Communications workshops.

https://doi.org/10.1109/PIMRC.2014.7136299

All material supplied via JYX is protected by copyright and other intellectual property rights, and duplication or sale of all or part of any of the repository collections is not permitted, except that material may be duplicated by you for your research use or educational purposes in electronic or print form. You must obtain permission for any other use. Electronic or print copies may not be offered, whether for sale or otherwise to anyone who is not an authorised user. 


\title{
Extension of Deflection Coefficient for Linear Fusion of Quantized Reports in Cooperative Sensing
}

\author{
Younes Abdi and Tapani Ristaniemi \\ Faculty of Information Technology, University of Jyväskylä, FIN-40014 Jyväskylä, Finland \\ Email: younes.abdi@jyu.fi, tapani.ristaniemi@jyu.fi
}

\begin{abstract}
Maximizing the so-called deflection coefficient is commonly used as an effective approach to design cooperative sensing schemes with low computational complexity. In this paper, an extension to the deflection coefficient is proposed which captures the effects of the quantization processes at the sensing nodes, jointly with the impact of linear combining at the fusion center. The proposed parameter is then used to formulate a new mixed-integer nonlinear programming problem as a fast suboptimal method to design a distributed detection scenario where the nodes report their sensing outcomes to a fusion center through nonideal digital links. Numerical evaluations show that the performance of the proposed method is very close to the optimal case.
\end{abstract}

\section{INTRODUCTION}

Spectrum sensing is the key element in each cognitive radio (CR) system and enables its user, commonly referred to as secondary user (SU), to find transmission opportunities in spectrum resources allocated exclusively to license holders. In this context, the license holders are called primary users (PU) and have the exclusive right of using the spectrum. The reliability of spectrum sensing is greatly enhanced through establishing certain kinds of cooperation among the sensing nodes. This cooperation is commonly coordinated by and the overall sensing outcome is generated in a special node called the fusion center (FC). Specifically, each node first performs spectrum sensing individually by using its own builtin sensing scheme. Then, the sensing nodes send their local sensing outcomes to the FC through the so-called reporting channels and finally, the FC combines the received local sensing outcomes to decide the presence or absence of the PU.

It is worth noting that, for a distributed detection problem with nonideal analogue communication channels between the distributed nodes and FC, the globally optimal structure is to perform likelihood ratio test (LRT) both at individual nodes and at the FC [1]. However, how to efficiently find the optimal LRT thresholds for individual nodes and for the fusion center is still unknown [2]. For the quantized soft decision case, i.e., when the reporting is performed through nonideal (i.e., erroneous) digital links, a solution for optimizing the local quantization levels jointly with the LRT threshold at the FC may or may not exist [3]. Even if the optimal solution exists, the threshold calculations are not trivial and complex optimization schemes are needed to solve them. These difficulties are commonly avoided by assuming a linear fusion scheme [2], [4]-[7] which is the base for our considered architecture.
In particular, linear combining is shown in [2] to perform very closely to the optimal LRT method with much less computational complexity.

Maximizing the so-called deflection coefficient (DC) [8], [9] or its modified version, modified deflection coefficient (MDC) [2], [4], [6], [7] is commonly used in the literature as a fast suboptimal approach to design effective fusion schemes in distributed detection scenarios. Using this parameter, i.e., the variance-normalized distance between the centers of two conditional distributions of the global test summary, is effective in the sense that it provides very close results to the ones obtained by the optimal LRT method at low computational cost. This method is of special interest when direct formulation of the false alarm and missed detection probabilities leads to nonconvex optimization problems.

In this paper, we assume that the FC performs linear combining on the reported local test summaries which receive through nonideal digital reporting channels. We first extend our previous analysis in [6] and [7] to the case in which the CR nodes use analogue-to-digital converters (ADC) with non-integer bit resolutions. Then, we propose a new version of the deflection coefficient which captures the effects of the quantization process at the $\mathrm{CR}$ nodes jointly with the linear fusion at the FC. Through these extensions, we construct and solve a new mixed-integer nonlinear programming problem to optimize the linear combining process at the FC, jointly with the number of levels used by each node for quantizing the sensing outcomes before reporting them.

\section{SySTEM MODEL}

A cognitive radio network ( $\mathrm{CRN})$ with $K$ sensing nodes is considered in this paper. These nodes cooperatively sense the radio spectrum to find temporal and/or spatial vacant bands for their data communication. In our adopted model, the $k$ th sample of the received PU signal at the $i$ th $\mathrm{CR}$ node is represented as

$$
\left\{\begin{aligned}
x_{i}(k) & =\nu_{i}(k), & & \mathcal{H}_{0} \\
x_{i}(k) & =h_{i} s(k)+\nu_{i}(k), & & \mathcal{H}_{1}
\end{aligned}\right.
$$

where $\mathcal{H}_{1}$ and $\mathcal{H}_{0}$ denote the hypotheses representing the presence or absence of the PU, respectively. $s(k)$ denotes the signal transmitted by the PU and $x_{i}(k)$ is the received signal by the the $i$ th $\mathrm{SU} . h_{i}$ is the listening channel block fading gain and $\nu_{i}(k) \sim \mathcal{C N}\left(0, \sigma_{\nu_{i}}^{2}\right)$ denotes the additive white Gaussian noise (AWGN). Without loss of generality, $s(k)$ and 
$\left\{\nu_{i}(k)\right\}$ are assumed to be independent of each other. CR node $i, i=1, \ldots, K$ performs spectrum sensing using its builtin sensor (which can be of any common types like Energy Detection (ED), Cyclostationary Detection (CSD), etc.) to derive a local test statistic $u_{i}$ and then uses the following quantization rule to map it on a bit sequence of length $d_{i}$

$$
\psi_{i}\left(u_{i}\right)=q_{n, i} \quad \text { if } \quad t_{n, i} \leqslant u_{i}<t_{n+1, i}
$$

where $\psi_{i}(\cdot)$ denotes the quantization process at the $i$ th SU, $q_{n, i}, n=1, \ldots, 2^{d_{i}}$ is its $n$th quantization level, while $t_{n, i}$ and $t_{n+1, i}$ denote the corresponding boundaries. Given the local sensing method and the quantization processes incorporated at the $i$ th sensing node, the probability distribution of $\psi_{i}$ is obtained. We denote the number of quantization bits used in all sensing nodes by $\mathbf{d} \triangleq\left[d_{1}, \ldots, d_{K}\right]^{T}$.

The generated reporting bit sequences are then transmitted to the FC through the reporting channel in an orthogonal manner. The effect of reporting channel impairments on the transmitted bit sequences of the $i$ th $\mathrm{CR}$ node is modeled as a bit error probability (BEP) denoted by $P_{\mathrm{b}, i}$. The reporting channel is assumed to affect each node's transmitted reporting bit sequence independently. Moreover, errors introduced on different bits in a transmitted reporting sequence by the reporting channel are assumed to be independent and identically distributed (i.i.d). Therefore, the received quantized test statistics at the $\mathrm{FC}, y_{i}, i=1, \ldots, K$, are independent discrete random variables whose probability mass functions (pmf) can be represented (for $j=0,1$ ) as [3]

$$
\begin{aligned}
& \operatorname{Pr}\left\{y_{i}=q_{n, i} \mid \mathcal{H}_{j}\right\}= \\
& \quad \sum_{k=1}^{2^{d_{i}}} P_{\mathrm{b}, i}^{D_{n, k}}\left(1-P_{\mathrm{b}, i}\right)^{d_{i}-D_{n, k}} \operatorname{Pr}\left\{\psi_{i}\left(u_{i}\right)=q_{k, i} \mid \mathcal{H}_{j}\right\}
\end{aligned}
$$

where $D_{n, k}$ is the Hamming distance between bit sequences corresponding to levels $q_{n, i}$ and $q_{k, i}$.

Focusing on transmitted and received bit strings in the reporting phase, we model the effect of the reporting channel by using the Exclusive OR (XOR) operator as $r_{i}=s_{i} \oplus e_{i}$ where $d_{i}$-bit random variables $s_{i}, r_{i}$, and $e_{i}$ denote the sent and received bit sequences and error caused by the reporting channel, respectively. If we denote the value of $s_{i}$ associated with the $n$th quantization level (i.e., $q_{n, i}$ ) by $s_{n, i}$, the following invertible mapping describes the correspondence between the quantization levels and the bit sequences

$$
\left\{\begin{aligned}
\Gamma:\left\{1,2, \ldots, 2^{d_{i}}\right\} & \rightarrow\left\{0,1, \ldots, 2^{d_{i}}-1\right\} \\
s_{n, i} & =\Gamma(n)
\end{aligned}\right.
$$

In other words, $s_{i}=\Gamma(n)$ if and only if $\psi_{i}=q_{n, i}$, or equivalently, $s_{i}=n$ if and only if $\psi_{i}=q_{\Gamma^{-1}(n), i}$. Therefore, the pmf of $s_{i}$ can be expressed as (for $n=0,1, \ldots, 2^{d_{i}}-1$, and $j=0,1)$

$$
P_{s_{i} \mid \mathcal{H}_{j}}(n) \triangleq \operatorname{Pr}\left\{s_{i}=n \mid \mathcal{H}_{j}\right\}=\operatorname{Pr}\left\{\psi_{i}=q_{\Gamma^{-1}(n), i} \mid \mathcal{H}_{j}\right\} .
$$

Without loss of generality, we have assumed the same mapping process for all CR nodes.
Given the BEP $P_{b, i}$, each bit in the random variable $e_{i}$ follows the Bernoulli distribution. Consequently, the pmf of $e_{i}$ is derived as, (for $n=0,1, \ldots, 2^{d_{i}}-1$ )

$$
P_{e_{i}}(n)=P_{b, i}^{w_{H}(n)}\left(1-P_{b, i}\right)^{d_{i}-w_{H}(n)}
$$

where $w_{H}(n)$ denotes the Hamming weight of the binary representation of $n$.

In order to derive the pmf of $r_{i}$, we use the fact that the assumed reporting channel contamination does not depend on the reported bit sequence $s_{i}$, nor the behavior of the PU, i.e., [7]

$$
\begin{array}{r}
P_{r_{i} \mid \mathcal{H}_{j}}(n)=\sum_{k=0}^{2^{d_{i}}-1} \operatorname{Pr}\left\{r_{i}=n\left|e_{i}=k\right| \mathcal{H}_{j}\right\} \operatorname{Pr}\left\{e_{i}=k\right\} \\
=\sum_{k=0}^{2^{d_{i}}-1} \operatorname{Pr}\left\{s_{i}=n \oplus k\left|e_{i}=k\right| \mathcal{H}_{j}\right\} \operatorname{Pr}\left\{e_{i}=k\right\} \\
=\sum_{k=0}^{2^{d_{i}}-1} \operatorname{Pr}\left\{s_{i}=n \oplus k \mid \mathcal{H}_{j}\right\} \operatorname{Pr}\left\{e_{i}=k\right\} \\
=\sum_{k=0}^{2^{d_{i}}-1} P_{s_{i} \mid \mathcal{H}_{j}}(n \oplus k) P_{b, i}^{w_{H}(k)}\left(1-P_{b, i}\right)^{d_{i}-w_{H}(k)}
\end{array}
$$

Assuming a general M-ary modulation for the reporting channel, the reporting BEP can be expressed as

$$
P_{\mathrm{b}, i}=c_{M} Q\left(\sqrt{\frac{c_{M}^{\prime \prime}}{d_{i}}}\right)
$$

where $c_{M}^{\prime \prime}=\frac{c_{M}^{\prime}\left|h_{r, i}\right|^{2} E_{r}}{N_{0} \log _{2} M}, Q(x) \triangleq \int_{x}^{\infty} \exp \left(-t^{2} / 2\right) d t / \sqrt{2 \pi}$ is the Q-function, $c_{M}$ and $c_{M}^{\prime}$ are two constants determined by the modulation type. $h_{\mathrm{r}, i}, E_{\mathrm{r}}$, and $N_{0}$ denote the reporting channel gain, reporting signal energy, and noise power spectral density, respectively.

Linear combining is performed at the FC, meaning that, the global test statistic $y_{c}$ is constructed as a weighted sum of the received quantized levels, i.e., $y_{\mathrm{c}}=\mathbf{w}^{T} \mathbf{y}$ where $\mathbf{w} \triangleq$ $\left[w_{1}, \ldots, w_{K}\right]^{T}$ and $\mathbf{y} \triangleq\left[y_{1}, \ldots, y_{K}\right]^{T}$. Finally, $y_{c}$ is compared against a predefined threshold $\xi$ to decide the presence or absence of the PU, i.e.,

$$
\begin{cases}\mathcal{H}_{1}, & y_{\mathrm{c}} \geq \xi \\ \mathcal{H}_{0}, & y_{\mathrm{c}}<\xi\end{cases}
$$

The detector performance is commonly measured using two probabilities, namely the probability of false alarm $P_{\mathrm{fa}}=$ $\operatorname{Pr}\left\{y_{\mathrm{c}} \geq \xi \mid \mathcal{H}_{0}\right\}$ and the probability of missed detection $P_{\mathrm{md}}=$ $\operatorname{Pr}\left\{y_{\mathrm{c}}<\xi \mid \mathcal{H}_{1}\right\}$. Both false alarm and missed detection probabilities depend on the probability distribution of the global test statistics $y_{c}$ which can be derived as a convolution of the pmfs of $K$ independent random variables $\left\{y_{i}\right\}_{i=1}^{K}$.

\section{REPORTING-FUSION OPTIMIZATION}

Our goal is to jointly optimize $\mathbf{w}$ and $\mathbf{d}$ to achieve the best cooperative sensing performance. We determine the weighting vector $\mathbf{w}$ at the FC and $\mathbf{d}$ used by the sensing nodes, through 
jointly considering the effects of both the listening and reporting channels.

We first formulate the optimization problem based on minimizing the missed detection probability subject to an upper bound on the false alarm probability

$$
\begin{array}{ll} 
& \min _{\mathbf{w}, \mathbf{d}} P_{\mathrm{md}} \quad(\mathrm{P} 1) \\
\text { s.t. } & P_{\mathrm{fa}} \leq \alpha
\end{array}
$$

where $\alpha$ is the given upper limit on the false alarm probability.

According to the central limit theorem, if $K$ is large enough, we can assume a Gaussian distribution for $y_{c}$ and the false alarm and missed detection probabilities can be expressed in closed form as

$$
\begin{array}{r}
P_{\mathrm{fa}}=Q\left(\frac{\xi-\boldsymbol{\mu}_{\mathcal{H}_{0}}^{T} \mathbf{w}}{\sqrt{\mathbf{w}^{T} \boldsymbol{\Sigma}_{\mathcal{H}_{0}} \mathbf{w}}}\right) \\
P_{\mathrm{md}}=1-Q\left(\frac{\xi-\boldsymbol{\mu}_{\mathcal{H}_{1}}^{T} \mathbf{w}}{\sqrt{\mathbf{w}^{T} \boldsymbol{\Sigma}_{\mathcal{H}_{1}} \mathbf{w}}}\right)
\end{array}
$$

where (for $j=1,2) \boldsymbol{\mu}_{\mathcal{H}_{j}} \triangleq \mathrm{E}\left[\mathbf{y} \mid \mathcal{H}_{j}\right]$ and $\boldsymbol{\Sigma}_{\mathcal{H}_{j}} \triangleq$ $\mathrm{E}\left[\mathbf{y} \mathbf{y}^{T} \mid \mathcal{H}_{j}\right]=\operatorname{diag}\left(\sigma_{y_{1} \mid \mathcal{H}_{j}}^{2}, \ldots, \sigma_{y_{K} \mid \mathcal{H}_{j}}^{2}\right)$. We have found through numerical evaluations that Gaussian distribution fits well for $K \geq 5$. Now if we eliminate $\xi$ in Eqs. (10) and (11) by considering a target false alarm probability $P_{\mathrm{fa}}=\alpha$, (P1) is converted to

$$
\max _{\mathbf{w}, \mathbf{d}} Q\left(\frac{Q^{-1}(\alpha) \sqrt{\mathbf{w}^{T} \boldsymbol{\Sigma}_{\mathcal{H}_{0} \mathbf{w}}}-\mathbf{a}^{T} \mathbf{w}}{\sqrt{\mathbf{w}^{T} \boldsymbol{\Sigma}_{\mathcal{H}_{1}} \mathbf{w}}}\right)
$$

where $Q^{-1}(\cdot)$ is the functional inverse of the Q-function, $\mathbf{a} \triangleq\left[a_{1}, \ldots, a_{K}\right]^{T} \triangleq \boldsymbol{\mu}_{\mathcal{H}_{1}}-\boldsymbol{\mu}_{\mathcal{H}_{0}}$ and for $i=1, \ldots, K$ we have $a_{i} \triangleq \mathrm{E}\left[y_{i} \mid \mathcal{H}_{1}\right]-\mathrm{E}\left[y_{i} \mid \mathcal{H}_{0}\right]$. In our previous work [7], we have developed a Branch-and-Bound $(\mathrm{BnB})$ procedure to solve (P2). Moreover, we have shown in [7] that the statistics of the reported quantized test summaries, i.e., a, $\boldsymbol{\Sigma}_{\mathcal{H}_{0}}$, and $\boldsymbol{\Sigma}_{\mathcal{H}_{1}}$ can be obtained by the following relation

$$
E\left[y_{i}^{\lambda} \mid \mathcal{H}_{j}\right]=\sum_{n=1}^{2^{d_{i}}} q_{n, i}^{\lambda} P_{r_{i} \mid \mathcal{H}_{j}}(\Gamma(n)), \quad \lambda=1,2, \ldots
$$

where $P_{r_{i} \mid \mathcal{H}_{j}}(n)$ can be expressed as a function of $d_{i}$ as

$$
\begin{array}{r}
P_{r_{i} \mid \mathcal{H}_{j}}(n)=\left(1-P_{b, i}\right)^{d_{i}} P_{s_{i} \mid \mathcal{H}_{j}}(n) \\
+\sum_{n_{e}=1}^{d_{i}} P_{b, i}^{n_{e}}\left(1-P_{b, i}\right)^{d_{i}-n_{e}} \sum_{k_{1}=1}^{d_{i}} \sum_{\substack{k_{2}=1 \\
k_{2} \neq k_{1}}}^{d_{i}} \cdots \\
\sum_{\substack{k_{n_{e}}=1 \\
k_{n_{e}} \neq k_{1}, \ldots, k_{n_{e}-1}}}^{d_{i}} P_{s_{i} \mid \mathcal{H}_{j}}\left(g\left(n, k_{1}, k_{2}, \ldots, k_{n_{e}}\right)\right)
\end{array}
$$

and

$$
\begin{aligned}
g\left(n, k_{1}, k_{2}, \ldots, k_{n_{e}}\right) \triangleq & n+\left[2 u\left(0.5-b_{k_{1}}(n)\right)-1\right] 2^{k_{1}-1} \\
+ & {\left[2 u\left(0.5-b_{k_{2}}(n)\right)-1\right] 2^{k_{2}-1}+\ldots } \\
& +\left[2 u\left(0.5-b_{k_{n_{e}}}(n)\right)-1\right] 2^{k_{n_{e}}-1}
\end{aligned}
$$

where $k_{i}, i=1, \ldots, n_{e}$ denotes the location of $i$ th 1 in $k$, $b_{j}(n), j=1, \ldots, d_{i}$ denotes the value of $j$ th bit in $n$ and $u(\cdot)$ is the step function which equals to 1 when its argument is positive and 0 otherwise.

For simplicity, we have considered discrete values for $d_{i}$ so far. However, the number of quantization levels in ADCs is commonly characterized by their so-called bit resolution which is not necessarily an integer. For instance, one can use a 5.32-bit ADC to quantize a signal to 40 levels. Therefore, we extend the proposed analysis to account for non-integer values of $d_{i}$. We formally represent this matter by first modifying the mapping process as

$$
\left\{\begin{aligned}
\Gamma:\left\{1,2, \ldots, 2^{d_{i}}\right\} \rightarrow & \left\{0,1, \ldots, 2^{\left\lceil d_{i}\right\rceil}-1\right\} \\
s_{n, i} & =\Gamma(n)
\end{aligned}\right.
$$

where $\left\lceil d_{i}\right\rceil$ denotes the smallest integer greater that or equal to $d_{i}$. Then, the definition for the pmf has to be extended to account for the redundant bit strings. Since they are not used, their probability mass equals zero, i.e.,

$$
P_{s_{i} \mid \mathcal{H}_{j}}(n)=0 \quad \text { for } n \in\left\{0, \ldots, 2^{\left\lceil d_{i}\right\rceil}-1\right\}-\mathcal{R}_{\Gamma}
$$

where $\mathcal{R}_{\Gamma}$ denotes the range of the mapping process $\Gamma$. Finally, the pmf of the received bit strings is modified as

$$
\begin{array}{r}
P_{r_{i} \mid \mathcal{H}_{j}}(n)=\left(1-P_{b, i}\right)^{\left\lceil d_{i}\right\rceil} P_{s_{i} \mid \mathcal{H}_{j}}(n) \\
+\sum_{n_{e}=1}^{\left\lceil d_{i}\right\rceil} P_{b, i}^{n_{e}}\left(1-P_{b, i}\right)^{\left\lceil d_{i}\right\rceil-n_{e}} \sum_{k_{1}=1}^{\left\lceil d_{i}\right\rceil} \sum_{\substack{\left.k_{2}=1 \\
k_{2} \neq k_{1}\right\rceil}}^{\left\lceil d_{i}\right.} \ldots \\
\sum_{\substack{k_{n_{e}}=1 \\
k_{n_{e}} \neq k_{1}, \ldots, k_{n_{e}-1}}}^{\left\lceil d_{i}\right\rceil} P_{s_{i} \mid \mathcal{H}_{j}}\left(g\left(n, k_{1}, k_{2}, \ldots, k_{n_{e}}\right)\right)
\end{array}
$$

The optimal linear fusion of analogue sensing outcomes can be derived through considering the Lagrange dual problem and Karush-Kuhn-Tucker (KKT) conditions [5]. Similarly, for a given $\mathbf{d}$, (P2) can be solved for optimal weighting vector as

$$
\tilde{\mathbf{w}}=\boldsymbol{\Sigma}_{\mathcal{H}_{0}}^{-1 / 2}\left[Q^{-1}(\alpha) \mathbf{I}_{K}+\zeta \mathbf{A}\right]^{-1} \mathbf{c}
$$

where $\mathbf{A} \triangleq \boldsymbol{\Sigma}_{\mathcal{H}_{1}} \boldsymbol{\Sigma}_{\mathcal{H}_{0}}^{-1}$ and $\mathbf{c} \triangleq \boldsymbol{\Sigma}_{\mathcal{H}_{0}}^{-1 / 2}$ a. $\zeta$ is the single root of the polynomial equation

$$
\left\|\left[Q^{-1}(\alpha) \mathbf{I}_{K}+\zeta \mathbf{A}\right]^{-1} \mathbf{c}\right\|=1
$$

and satisfies

$$
Q^{-1}(\alpha) \mathbf{I}_{K}+\zeta \mathbf{A} \succ \mathbf{0}
$$

where $\mathbf{0}$ stands for the null matrix and $\succ$ represents the element-wise inequality. Note that (19) and (20) specify $\zeta$ as a function of $\mathbf{d}$.

In order to gain insight about the major elements in (P2), we rewrite it in the following form by using (18) and through some basic algebraic manipulations,

$$
\min _{\mathbf{d}, \zeta} \varphi(\mathbf{d}, \zeta)
$$


where

$$
\varphi(\mathbf{d}, \zeta) \triangleq \frac{Q^{-1}(\alpha)-\sum_{i=1}^{K} \frac{a_{i}^{2}}{Q^{-1}(\alpha) \sigma_{y_{i} \mid \mathcal{H}_{0}}^{2}+\zeta \sigma_{y_{i} \mid \mathcal{H}_{1}}^{2}}}{\sqrt{\sum_{i=1}^{K} \frac{a_{i}^{2} \sigma_{y_{i} \mid \mathcal{H}_{1}}^{2}}{\left(Q^{-1}(\alpha) \sigma_{y_{i} \mid \mathcal{H}_{0}}^{2}+\zeta \sigma_{y_{i} \mid \mathcal{H}_{1}}^{2}\right)^{2}}}}
$$

Since the Q-function is strictly decreasing with respect to its argument, we have removed it from (P3) and turned the problem into a minimization.

It is worth noting that, the local sensing outcomes have greater variances when $\mathcal{H}_{1}$ is true (i.e., when the $\mathrm{PU}$ is present), compared to $\mathcal{H}_{0}$ case. Since the local sensing outcomes pass through the same quantization and reporting processes to reach the FC-regardless of whether the PU is active or not - the received sensing outcomes at the FC have greater variances in general when the PU signal is present. That is, $\sigma_{y_{i} \mid \mathcal{H}_{1}}^{2} \geq \sigma_{y_{i} \mid \mathcal{H}_{0}}^{2}$. Hence, we have

$$
\tilde{\mathbf{w}}^{T} \boldsymbol{\Sigma}_{\mathcal{H}_{1}} \tilde{\mathbf{w}} \geq \tilde{\mathbf{w}}^{T} \boldsymbol{\Sigma}_{\mathcal{H}_{0}} \tilde{\mathbf{w}}=1
$$

Applying this inequality on our cost function $\varphi(\mathbf{d}, \zeta)$, we derive an upper bound, i.e.,

$$
\varphi(\mathbf{d}, \zeta) \leq Q^{-1}(\alpha)-\sum_{i=1}^{K} \frac{a_{i}^{2}}{Q^{-1}(\alpha) \sigma_{y_{i} \mid \mathcal{H}_{0}}^{2}+\zeta \sigma_{y_{i} \mid \mathcal{H}_{1}}^{2}}
$$

A closer look at (22) leads to some interesting observations. In fact, the term inside the summation is the ratio of two performance metrics. Specifically, the numerator $a_{i}^{2}$ measures the sensor ability to discriminate between $\mathcal{H}_{0}$ and $\mathcal{H}_{1}$, whereas the denominator, measures the sensor uncertainty in declaring either $\mathcal{H}_{0}$ or $\mathcal{H}_{1}$. Note that due to (20), the denominator in (22) represents a positive linear combination of reported test summary variances $\sigma_{y_{i} \mid \mathcal{H}_{0}}^{2}$ and $\sigma_{y_{i} \mid \mathcal{H}_{1}}^{2}$. Therefore, the ratio in (22) can be interpreted as the SNR (measuring the detection quality) of the $i$ th sensor report when received at the FC. In fact, it can be considered as an extended version of the DC and MDC which have been introduced in literature as good measures for performance optimization in cooperative spectrum sensing. We refer to the ratio in (22) as extended deflection coefficient (EDC).

These observations motivate us to propose a new approach for the discussed joint reporting-fusion optimization based on the EDC. We formally define EDC as

$$
\Delta_{\text {ext }}^{2} \triangleq \frac{\left(\mathrm{E}\left[y_{c} \mid \mathcal{H}_{1}\right]-\mathrm{E}\left[y_{c} \mid \mathcal{H}_{0}\right]\right)^{2}}{Q^{-1}(\alpha) \operatorname{Var}\left\{y_{c} \mid \mathcal{H}_{0}\right\}+\zeta \operatorname{Var}\left\{y_{c} \mid \mathcal{H}_{1}\right\}}
$$

Replacing $y_{c}$ with its weighted sum definition, we have

$$
\Delta_{\mathrm{ext}}^{2}=\frac{\left(\mathbf{a}^{T} \mathbf{w}\right)^{2}}{\mathbf{w}^{T}\left[Q^{-1}(\alpha) \boldsymbol{\Sigma}_{\mathcal{H}_{0}}+\zeta \boldsymbol{\Sigma}_{\mathcal{H}_{1}}\right] \mathbf{w}}
$$

We aim at finding $\mathbf{w}$ and $\mathbf{d}$ such that

$$
\begin{array}{ll} 
& \max _{\mathbf{w}, \mathbf{d}} \Delta_{\text {ext }}^{2}(\mathrm{P} 4) \\
\text { s.t. } & \|\mathbf{w}\|=1
\end{array}
$$

The constraint on the weight vector norm is necessary here to derive a unique solution since the EDC does not depend on $\|\mathbf{w}\|$. In order to derive an analytical solution for (P4) we first eliminate $\mathbf{w}$ as follows. Through the linear transformation [2]

$$
\mathbf{w}^{\prime}=\boldsymbol{\Sigma}_{\text {ext }}^{1 / 2} \mathbf{w}
$$

the EDC is converted to

$$
\Delta_{\mathrm{ext}}^{2}=\frac{\mathbf{w}^{\prime T} \boldsymbol{\Sigma}_{\mathrm{ext}}^{-T / 2} \mathbf{a a}^{T} \boldsymbol{\Sigma}_{\mathrm{ext}}^{-1 / 2} \mathbf{w}^{\prime}}{\mathbf{w}^{\prime T} \mathbf{w}^{\prime}} \leq\left\|\boldsymbol{\Sigma}_{\mathrm{ext}}^{-T / 2} \mathbf{a}\right\|^{2}
$$

where $\boldsymbol{\Sigma}_{\text {ext }} \triangleq Q^{-1}(\alpha) \boldsymbol{\Sigma}_{\mathcal{H}_{0}}+\zeta \boldsymbol{\Sigma}_{\mathcal{H}_{1}}$ and the inequality follows the Rayleigh-Ritz inequality. The equality is achieved when

$$
\mathbf{w}^{\prime}=\boldsymbol{\Sigma}_{\mathrm{ext}}^{-T / 2} \mathbf{a}
$$

Therefore, the optimal w which maximizes the EDC is derived as a function of $\mathbf{d}$ as

$$
\mathbf{w}_{\mathrm{edc}}=\frac{\boldsymbol{\Sigma}_{\mathrm{ext}}^{-1 / 2} \mathbf{w}^{\prime}}{\left\|\boldsymbol{\Sigma}_{\mathrm{ext}}^{-1 / 2} \mathbf{w}^{\prime}\right\|}
$$

Replacing $\mathbf{w}$ with its EDC-optimal value $\mathbf{w}_{\text {edc }}$, the EDC can be rewritten as a function of $\mathbf{d}$

$$
\Delta_{\text {ext }}^{2}=\mathbf{a}^{T} \boldsymbol{\Sigma}_{\text {ext }}^{-1} \mathbf{a}=\sum_{i=1}^{K} \frac{a_{i}^{2}}{Q^{-1}(\alpha) \sigma_{y_{i} \mid \mathcal{H}_{0}}^{2}+\zeta \sigma_{y_{i} \mid \mathcal{H}_{1}}^{2}}
$$

Now, by comparing (29) with (22) we see that maximizing the variance-normalized distance between the centers of two conditional distributions of the global test summary $y_{c}$ is equivalent to minimizing the upper bound in (22). Moreover, due to the existence of $\zeta$ in (29), (P4) is a MINLP problem which is NP-hard in general. A standard method for solving a MINLP is the BnB procedure. As mentioned before, we have developed a BnB algorithm in [7] for (P3) and a similar approach can be used to solve (P4) as well. Here, we develop an alternative suboptimal approach based on EDC which leads to nearly optimal performance, but at much lower computational cost. The proposed method is based on the fact that $\sigma_{y_{i} \mid \mathcal{H}_{0}}^{2}$ and $\sigma_{y_{i} \mid \mathcal{H}_{1}}^{2}$ only depend on $d_{i}$ and considering a fixed value for $\zeta$, converts (P4) from a $(K+1)$-dimensional problem into $K$ one-dimensional integer programs. In other words, the computational complexity of the optimization is drastically decreased by considering a fixed $\zeta$. Therefore, we decompose the optimization procedure into two consecutive processes in an iteration loop. Specifically, starting with an initial point for $\mathbf{d}$ denoted by $\mathbf{d}^{(0)}$, at $k$ th iteration, we seek the best $\mathbf{d}^{(k)}$ based on $\zeta^{(k-1)}$ by solving the following set of optimizations,

For $i=1, \ldots, K$,

$$
d_{i}^{(k)}=\underset{d_{i}}{\operatorname{argmax}} \frac{a_{i}^{2}}{Q^{-1}(\alpha) \sigma_{y_{i} \mid \mathcal{H}_{0}}^{2}+\zeta^{(k-1)} \sigma_{y_{i} \mid \mathcal{H}_{1}}^{2}}
$$

where $d_{i}^{(k)}$ and $\zeta^{(k)}$ denote the values of $d_{i}$ and $\zeta$ at $k$ th iteration, respectively. Then, through considering (19) and (20) we find $\zeta^{(k)}$ for $\mathbf{d}^{(k)}$. Consequently, at each iteration we are dealing with $K$ one-dimensional nonlinear integer programs. Therefore, the computational complexity of this approach increases linearly with the number of sensing nodes $K$. 
TABLE I

LOW-COMPLEXITY LINEAR FUSION OF QUANTIZED REPORTS IN COOPERATIVE SENSING

\begin{tabular}{|ll|}
\hline Input: & $\varepsilon$ \\
Output: & Suboptimal $\mathbf{d}, \mathbf{w}$ \\
\hline 1. & Initialize $\mathbf{d}$ as $\mathbf{d}^{(0)}$ \\
2. & Solve $(19)$ and $(20)$ for $\zeta^{(0)}$ \\
3. & $k \leftarrow 0 ;$ \\
4. & $\varphi^{(0)} \leftarrow \varphi\left(\mathbf{d}^{(0)}, \zeta^{(0)}\right) ;$ \\
5. & do \\
6. & $k \leftarrow k+1 ;$ \\
7. & Solve $(\mathrm{P} 5)$ for $\mathbf{d}^{(k)} ;$ \\
8. & Plug $\mathbf{d}^{(k)}$ into $(19)$ and $(20)$ and solve them for $\zeta^{(k)} ;$ \\
9. & $\varphi^{(k)} \leftarrow \varphi\left(\mathbf{d}^{(k)}, \zeta^{(k)}\right) ;$ \\
10. & while $\left|\varphi^{(k)}-\varphi^{(k-1)}\right| \geq \varepsilon ;$ \\
11. & return $\mathbf{d}^{(k)}$ and $\zeta^{(k)} ;$
\end{tabular}

Numerical evaluations indicate that the desired performance is achieved by only a few iterations. A pseudocode of the proposed algorithm is presented in Table I.

\section{Numerical Results}

The ED and uniform quantization have been adopted as local sensing and test summary quantization methods at the CR nodes, respectively. In all simulations, there are $K=5$ cooperating nodes which transmit their sensing outcomes over the reporting channels using the BPSK modulation. The PU signal is modeled as a direct-sequence spread-spectrum BPSK signal using Walsh-Hadamard code with length 16, i.e., processing gain of 16 are considered in all simulation results. The maximum number of quantization bits in each node is 7 .

Fig. 1 depicts the results derived as Complementary Receiver Operational Characteristics (CROC) curves. Specifically, three cases have been considered as: Case\#1) Depicts the performance of uniform linear combining at the fusion center and maximum number of quantization bits at the sensing nodes, Case\#2) Depicts the performance of optimal linear combining at the fusion center and maximum number of quantization bits at the sensing nodes, Case\#3) Depicts the performance of the proposed joint optimization, i.e., optimal linear combining at the fusion center and optimal number of quantization bits at the sensing nodes.

It is worth noting that, Case\#1, Case\#2, and Case\#3 represent the detector design without any optimization, only with optimal weighting, and with joint reporting-fusion optimization respectively. The plots clearly illustrate the effectiveness of our proposed detector in terms of lower false alarm and missed detection probabilities which are shown as CROC curves closer to the origin. Moreover, it can be observed that the achieved optimization results based on both the Gaussian approximation and EDC are in close agreement with each other. Note that the optimal linear combining based on the Gaussian approximation is known to provide nearly-optimal performance [2].

\section{CONCLUSION}

In this paper, an extension to the deflection coefficient has been proposed which captures the effects of the quantization

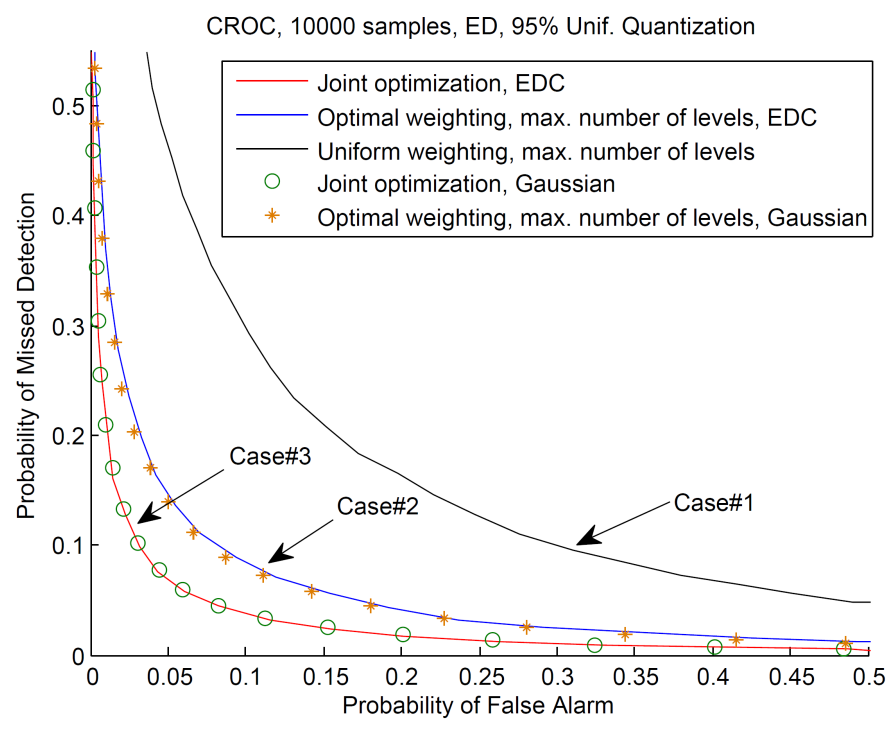

Fig. 1. CROC curves for the energy detection using 20 samples of the PU signal and uniform quantization with Chebyshev probability (see [7]) of $95 \%$. The listening channel SNR levels at sensor inputs are $\{0,-2.7,-3.1,-1.4,-6.9\}$ in $\mathrm{dB}$. The reporting channel SNR levels are $\{10,13,12,14,11\}$ in $\mathrm{dB}$. The results are obtained using 10,000 noise realizations.

processes at the sensing nodes, jointly with the impact of the linear combining at the fusion center. The proposed parameter has been used to formulate a new MINLP problem as a fast suboptimal method to design a distributed detection scenario where the nodes report their sensing outcomes to a fusion center through nonideal digital links. Numerical results demonstrate the effectiveness of the proposed design approach.

\section{REFERENCES}

[1] B. Chen and P. K. Willett, "On the optimality of the likelihood-ratio test for local sensor decision rules in the presence of nonideal channels," IEEE Trans. Inform. Theory, vol. 51, pp. 693-699, Feb. 2005.

[2] Z. Quan, S. Cui, and A. H. Sayed, "Optimal linear cooperation for spectrum sensing in cognitive radio networks," IEEE J. Sel. Topics Signal Process., vol. 2, no. 1, pp. 28-40, Feb. 2008.

[3] S. Chaudhari, J. Lunden, V. Koivunen, and H. V. Poor, "Cooperative sensing with imperfect reporting channels: Hard decisions or soft decisions?" IEEE Trans. Signal Process., vol. 60, no. 1, pp. 18-28, Jan. 2012.

[4] Z. Quan, S. Cui, A. H. Sayed, and H. V. Poor, "Optimal multiband joint detection for spectrum sensing in cognitive radio networks," IEEE Trans. Signal Process., vol. 57, no. 3, pp. 1128-1140, March 2009.

[5] G. Taricco, "Optimization of linear cooperative spectrum sensing for cognitive radio networks," IEEE J. Sel. Topics Signal Process., vol. 5, no. 1, pp. 77-86, Feb. 2011.

[6] Y. Abdi and T. Ristaniemi, "Joint reporting and linear fusion optimization in collaborative spectrum sensing for cognitive radio networks,", in Proc. 9th Intl. Conf. Inform., Commun., and Signal Process. (ICICS), Tainan, Taiwan, Dec. 2013, pp. 1-5.

[7] — , "Joint local quantization and linear cooperation in spectrum sensing for cognitive radio networks," IEEE Trans. Signal. Process., doi: 10.1109/TSP.2014.2330803.

[8] H. V. Poor, An introduction to signal detection and estimation. Berlin Heidelberg: Springer-Verlag, 1994.

[9] M. Derakhshani, T. Le-Ngoc, and M. Nasiri-Kenari, "Efficient cooperative cyclostationary spectrum sensing in cognitive radios at low SNR regimes," IEEE Trans. Wireless Commun., vol. 10, no. 11, pp. 3754-3764, Nov. 2011. 